1Universidade Federal de Pernambuco (UFPE) Recife (PE), Brasil. laisdesamenezes@gmail. com

2 Universidade Federal da Paraíba (UFPB) - João Pessoa (PB), Brasil.

\section{Estudo comparativo entre indicadores assistenciais de saúde bucal na Atenção Básica em Recife, Pernambuco}

\author{
Oral healthcare comparative study among indicators in Primary \\ Care, in Recife, Pernambuco
}

Laís de Sá Menezes' ${ }^{\mathbf{1}}$ Cindy Avani Silva Ceissler', Yuri Wanderley Cavalcanti², Nilcema

Figueiredo', Edson Hilan Gomes de Lucena²

DOI: 10.1590/0103-1104202112812

RESUMO A rede de atenção à Saúde Bucal (SB) se ampliou com a Política Nacional de Saúde Bucal, sendo os indicadores ferramentas para avaliar conquistas ou necessidade de mudanças. Este trabalho objetivou analisar indicadores de SB da Atenção Básica por tipo de organização das unidades de saúde em Recife, 2018. Trata-se de um estudo transversal, de natureza descritiva e analítica com base em dados secundários. Analisou-se a associação entre tipos de organização da Atenção Básica (Unidade de Saúde da Família - USF; Upinha e Unidade Básica Tradicional - UBT) e indicadores assistenciais de SB, através dos testes qui-quadrado e Kruskal-Wallis $(\mathrm{p}<0,05)$. A cobertura de primeira consulta odontológica e os procedimentos odontológicos per capita apresentaram mediana maior nas UBT, 31,8\% ( $p=0,00)$ e 0,6 $(\mathrm{p}=0,127)$, respectivamente. A proporção de exodontia e procedimentos clínicos foi maior nas USF com mediana $8,2 \%(\mathrm{p}=0,703)$. As USF foram as que realizaram o maior número de atividades coletivas de escovação dental supervisionada (45,3\%, p=0,082) e aplicação de flúor (44,6\%, p=0,174). Conclui-se que as UBT apresentaram melhores resultados nos indicadores relacionados ao acesso ao tratamento clínico e as USF realizaram mais ações coletivas de prevenção.

PALAVRAS-CHAVE Atenção Primária à Saúde. Saúde bucal. Modelos de assistência à saúde.

ABSTRACT The Oral Health care network $(\mathrm{OH})$ has expanded with the National Oral Health Policy, and the indicators are tools to assess achievements or the need for changes. This study aimed to analyze the $\mathrm{OH}$ indicators of Primary Care Basic by health units organization type in Recife, 2018. This is a cross-sectional, quantitative, descriptive and analytical study, based on secondary data. The association between organization type of Primary Care (Family Health Unity/USF; Upinha and Traditional Basic Health Unit/UBT) and $\mathrm{OH}$ assistance indicators was analyzed using the chi-square and Kruskal-Wallis tests $(p<0,05)$. The first dental appointment coverage and the average number of procedures per capita showed a higher median in the UBT, 31,8\% ( $p=0,00)$ and $0,6(p=0,127)$, respectively. The tooth extraction proportion was higher in the USF, with a median of $8,2 \%$ ( $p=0,703)$. The USF were the ones that performed the largest number of collective activities of supervised tooth brushing $(45.3 \%, p=0.082)$ and fluoride application $(44.6 \%, p=0.174)$. It is concluded that the UBT showed better results in the indicators related to access to clinical treatment and the USF performed more collective activities.

KEYWORDS Primary Health Care. Oral health. Healthcare models. 


\section{Introdução}

A Atenção Primária em Saúde (APS) é citada em 1920, no Relatório Dawson como uma das formas de organização do cuidado em saúde em níveis de atenção por meio dos chamados 'Centros de saúde primários'. Em 1978, na Declaração de Alma-Ata, a APS é apontada como o primeiro contato do sistema de saúde, devendo apresentar um olhar ampliado para o cuidado, agindo sob determinantes de saúde que vão além da genética, abarcando os aspectos sociais e físicos nos quais os usuários estão inseridos $\mathbf{1}$.

No Brasil, apesar de a APS apresentar aproximações com os princípios doutrinários do Sistema Único de Saúde (SUS), o seu desenvolvimento atribuiu-lhe outro significado. A incorporação de elementos do processo de redemocratização do País e de luta do movimento sanitário brasileiro distanciou-a do reducionismo conceitual e pautou a construção de um sistema público e universal em uma concepção de cidadania ampliada. Assim, para o SUS, em sua criação, optou-se pelo termo Atenção Básica $(\mathrm{AB})^{2}$.

No contexto brasileiro, propõe-se a Estratégia Saúde da Família (ESF), desde 1994, como programa prioritário de solidificação, expansão e reorientação da $\mathrm{AB}$, devendo garantir atenção integral ao usuário, com ações de vigilância em saúde, além de compreender e atuar sobre os múltiplos determinantes e condicionantes do processo saúde-doença ${ }^{3}$. A institucionalização da $\mathrm{AB}$ se deu por meio da Política Nacional da Atenção Básica (PNAB) em 2006, revisada em $2017^{4}$. Apesar de definir a ESF como estratégia prioritária, a última revisão incentiva outras formas de organização ao retomar o financiamento federal para $\mathrm{AB}$ tradicional, o que desfaz o compromisso com a expansão da ESF e com o SUS. Além disso, há a flexibilização da cobertura populacional e do número de Agentes Comunitários de Saúde (ACS), o que segmenta o cuidado, retomando a perspectiva seletiva da APS e favorecendo a desqualificação da AB brasileira ${ }^{5}$.
A inserção da saúde bucal só ocorreu sete anos após a criação da $\mathrm{ESF}^{6}$. No entanto, ainda de forma limitada, pois cada equipe de saúde bucal só podia atuar para cada duas ESF. Em 2004, com o Brasil Sorridente, Política Nacional de Saúde Bucal (PNSB), deu-se início à expansão de serviços de saúde bucal e à superação do ideário liberal-privativista, buscando uma reorientação do modelo de saúde bucal vigente. A PNSB orienta todos os níveis de atenção, fomentando ações multidisciplinares, intersetoriais e atividades nos âmbitos individual e coletivo de promoção da saúde, prevenção de doenças, diagnóstico, tratamento e reabilitação ${ }^{7-9}$. No âmbito da $A B$, essa política aponta para ampliação e qualificação, com estímulo ao vínculo com os usuários e superação do modelo biomédico, considerando a realidade local10.

Entretanto, ao longo do tempo, o número de equipes de Saúde da Família (eSF) mostra-se superior ao de equipes de Saúde Bucal (eSB). No Brasil, de 2002 a 2018, o número de eSF aumentou de 16.734 para 42.975, o que implicou uma expansão da cobertura de $31,9 \%$ para $64 \%$. Por outro lado, no período, para as eSB, o País apresentava 4.261 equipes e finalizou o recorte com 26.712 equipes, ampliando de $15 \%$ para $40 \%$ de usuários cobertos ${ }^{11}$. A mesma disparidade entre a cobertura de eSF e saúde bucal é observada em Pernambuco ${ }^{\mathbf{1 2}}$, e dados do Ministério da Saúde indicam que, em Recife, a cobertura de saúde bucal evoluiu de $19 \%$ para $34 \%$ no período de 2002 a 2018 , enquanto a de saúde da família obteve aumento de $30,7 \%$ para $55,9 \%{ }^{11}$.

Apesar dos avanços, a reorientação de um modelo assistencial não depende apenas da criação da política pública ${ }^{\mathbf{1 3}}$. Ainda existem desafios no trabalho em equipe, multidisciplinar e interprofissional, além de necessidade de avanços no cuidado integral ao paciente e na formação profissional voltada para o SUS ${ }^{14,15}$. Lima, Antunes e Silva ${ }^{16}$ destacam, também, o escasso conhecimento dos gestores de unidades de saúde sobre os Sistemas de Informação em Saúde (SIS) e a subutilização 
de indicadores de saúde para o planejamento e o controle dos serviços de saúde. Os indicadores de saúde são medidas-síntese baseadas em evidências que apresentam informações importantes sobre a situação sanitária e o desempenho dos sistemas de saúde, o que permite a vigilância das condições de saúde, sendo, portanto, uma poderosa ferramenta para a gestão e a avaliação da situação da saúde em todos os níveis ${ }^{\mathbf{1 7}}$.

A análise dos SIS e dos indicadores de saúde pode auxiliar na concepção de estratégias de aperfeiçoamento da gestão em saúde bucal. Bulgareli et al. ${ }^{18}$ e Magri et al. ${ }^{19}$ usam o indicador de resolutividade em saúde bucal para comparar a efetividade dos modelos de atenção à saúde na ESF e na AB tradicional e usam os indicadores para subsidiar a tomada de decisão pautada na realidade local.

Em Recife, o modelo de atenção à saúde bucal na $A B$ é dividido em três tipos de Unidades Básicas de Saúde (UBS): Unidades de Saúde da Família (USF) e Upinhas, ambas inseridas na ESF; e Unidades Básicas Tradicionais (UBT), que seguem o modelo tradicional sem divisão territorial ou cadastro de usuários. As USF seguem as normativas da Portaria do Ministério da Saúde n ${ }^{0}$ 2436/2017, com o funcionamento de 40 horas semanais, devendo ser compostas minimamente por médico, enfermeiro, auxiliar e/ou técnico de enfermagem e ACS. Podem fazer, também, parte da composição os agentes de combate às endemias e os profissionais de saúde bucal (cirurgião-dentista, auxiliar ou técnico em saúde bucal) ${ }^{4}$. As Upinhas foram instituídas em 2013 pela gestão municipal como um novo modelo de USF ${ }^{20}$ e destacam-se, para o modelo de atenção à saúde bucal, por apresentarem quantitativo equiparado de eSF e eSB. Além disso, apresentam duas modalidades de horário de funcionamento: quando Upinha 24 horas, o atendimento ambulatorial ocorre das 07 às 19 horas; quando Upinha Dia, segue o horário das USF convencionais. Já as UBT são mais antigas e estão em policlínicas ou Centros de Saúde, não havendo composição mínima de profissionais, nem ACS, e atendem a população descoberta pela ESF.

Dessa forma, observam-se diferenças estruturais, de funcionamento e de processo de trabalho nos tipos de organizações da $\mathrm{AB}$ do Recife. Assim, este trabalho objetivou analisar indicadores assistenciais de saúde bucal da $\mathrm{AB}$ com relação ao tipo de organização das unidades básicas de saúde na cidade de Recife/ Pernambuco, no ano de 2018.

\section{Material e métodos}

Trata-se de um estudo transversal, de natureza descritiva e analítica, realizado com dados secundários extraídos dos relatórios do Sistema de Informação em Saúde para a Atenção Básica (Sisab), referentes às eSB de UBS de Recife (PE), em 2018. No referido ano, Recife apresentava uma rede de $\mathrm{AB}$ de 138 UBS com atenção à saúde bucal. Para o modelo de ESF, eram 117 unidades com $172 \mathrm{eSB}$, que, de acordo com a gestão municipal, representam uma cobertura de 37\% da população. Destas unidades, 106 eram USF e continham o total de 144 eSB; as demais eram quatro Upinhas 24 horas e sete Upinhas Dia, totalizando 28 eSB. Para o modelo tradicional, o município apresentava 21 UBT com atendimento odontológico.

Na pesquisa, foram incluídas todas as UBS com oferta de saúde bucal cujo registro de informações no Sisab permitisse o cálculo dos indicadores do estudo, excluindo as que não possuíam cirurgião-dentista no registro do Cadastro Nacional de Estabelecimentos de Saúde (CNES).

A partir do tipo de organização das eSB da AB (USF, Upinha e UBT), verificou-se o resultado de cinco indicadores assistenciais de saúde bucal à $\mathrm{AB}$ : cobertura da primeira consulta odontológica programática, proporção de exodontia entre procedimentos clínicos odontológicos individuais selecionados, média de procedimentos odontológicos básicos individuais per capita na população, ação coletiva de aplicação tópica de flúor gel e ação coletiva de escovação dental supervisionada. 
Para o cálculo dos indicadores foram coletados dados referentes ao número de usuários cadastrados para cada UBT e eSB das USF e Upinhas e ao número de procedimentos realizados especificamente para cada índice. Os dados referentes à população cadastrada foram gerados por meio de relatórios consolidados do cadastro individual no e-SUS Atenção Básica (e-SUS AB), ajustando a data para 31 de dezembro de 2018, obtendo-se, assim, o número de 'cidadãos ativos' no relatório de cadastro individual. Ressalta-se que, por não existir um registro exato de pessoas atendidas pelas UBT, esses dados foram estimados com base na população cadastrada na Estratégia de Agentes Comunitários de Saúde (EACS). Todavia, diante da fragilidade desse dado em algumas UBS, quando o contingente populacional registrado foi menor do que o estabelecido na $\mathrm{PNAB}^{4}$, considerou-se o mínimo de 2.000 usuários para um cirurgião-dentista com carga horária de 40 horas, ou a soma das horas de trabalho semanais de um ou mais profissionais até se atingir a soma de 40 horas.

Os dados do número de procedimentos realizados referentes às USF e Upinhas foram obtidos com a Coordenação de Informação e Avaliação da AB da Prefeitura do Recife através dos relatórios gerenciais do Sisab, que têm como fonte a Coleta de Dados Simplificada (CDS) e o Prontuário Eletrônico do Cidadão (PEC), por meio do e-SUS AB. Já os dados referentes às UBT foram oriundos de endereço eletrônico de acesso público (http:// tabnet.saude.pe.gov.br/), que tem como fonte os Boletins de Produção Ambulatorial Consolidados (BPA-C) do Sistema de Informação Ambulatorial (SIA/SUS).

A partir do consolidado calcularam-se para cada eSB (USF e Upinha) e UBT os cinco indicadores investigados no estudo, conforme o quadro 1.

Quadro 1. Quadro resumo com os indicadores de saúde bucal utilizados no estudo, método de cálculo e procedimentos incluídos

\begin{tabular}{ll}
\hline Indicador & Método de cálculo \\
\hline $\begin{array}{l}\text { Cobertura da primeira } \\
\text { consulta odontológica } \\
\text { programática }\end{array}$ & $\begin{array}{l}\text { Número de primeiras consultas odontológicas } \\
\text { programáticas realizadas em determinado loc } \\
\text { e período dividido pela população total no me } \\
\text { mo local e período, multiplicado por } 100\end{array}$ \\
$\begin{array}{l}\text { Proporção de exodontia } \\
\text { entre procedimentos } \\
\text { clínicos odontológicos }\end{array}$ & $\begin{array}{l}\text { nente em determinado município e período } \\
\text { individuais selecionados }\end{array}$ \\
& $\begin{array}{l}\text { clínicos individuais no mesmo local e período, } \\
\text { multiplicado por } 100\end{array}$
\end{tabular}

Média de procedimentos odontológicos básicos individuais per capita
Procedimentos odontológicos básicos, clínicos e/ou cirúrgicos dividido pela população de determinado local
Procedimentos inclusos

Primeira consulta odontológica programática.

\section{.}

Exodontia de dente permanente, aplicação de cariostático, aplicação de selante, aplicação tópica de flúor, selamento provisório de cavidade dentária, capeamento pulpar, restauração de dente permanente anterior, restauração de dente permanente posterior, acesso à polpa dentária e medicação, curativo de demora com ou sem preparo biomecânico, pulpotomia dentária, raspagem alisamento e polimento supragengivais (por sextante) e raspagem alisamento subgengivais (por sextante).

Exodontia de dente permanente, aplicação de cariostático, aplicação de selante, aplicação tópica de flúor, selamento provisório de cavidade dentária, capeamento pulpar, restauração de dente permanente anterior, restauração de dente permanente posterior, acesso à polpa dentária e medicação, curativo de demora com ou sem preparo biomecânico, pulpotomia dentária, raspagem alisamento e polimento supragengivais (por sextante) e raspagem alisamento subgengivais (por sextante).

Ação coletiva de escovação dental supervisionada

Atividade realizada ou não

Ação coletiva de aplicação Atividade realizada ou não tópica de flúor
Escovação dental supervisionada.

Aplicação tópica de flúor. 
O indicador de cobertura da primeira consulta odontológica programática é um indicador de acesso e continuidade do cuidado, em que a primeira consulta odontológica programática refere-se à avaliação das condições gerais de saúde e realizaçã o de exame clínico odontológico com finalidade de diagnóstico e elaboração de um plano preventivo e terapêutico ${ }^{21}$.

$\mathrm{O}$ indicador de proporção de exodontia entre procedimentos clínicos odontológicos individuais selecionados demonstra o tipo de cuidado (mutilador/curativo) ofertado no serviço odontológico ${ }^{23}$. Quanto maior seu valor, maior percentual de extrações dentárias em comparação às ações preventivas e curativas ${ }^{24}$.

A média de procedimentos odontológicos básicos individuais per capita mede a resposta dos serviços odontológicos da $\mathrm{AB}$ às necessidades de assistência odontológica. Representa o número médio de procedimentos odontológicos para cada indivíduo em um determinado local e período ${ }^{25,26}$.

As ações coletivas em saúde bucal foram investigadas através dos indicadores de escovação dental supervisionada e aplicação tópica de flúor. O indicador escovação dental supervisionada corresponde à média do número de atividades de escovação dental com ou sem evidenciação de placa bacteriana sob a supervisão de um ou mais profissionais de saúde ${ }^{21}$, avalia efetividade na $\mathrm{AB}$ e dá noção de acesso a orientações para prevenção de doenças bucais ${ }^{24}$. Já a ação coletiva de aplicação tópica de flúor é realizada em grupos populacionais sob supervisão de um ou mais profissionais de saúde, aplicando-se flúor em gel na concentração de $1,23 \%$, utilizando escova dental, moldeira, entre outras formas de aplicação registrada por pessoa e por mês $\mathbf{~}^{\mathbf{2 1}}$.

Para análise dos indicadores de atividades coletivas, foi avaliada sua ocorrência ou não, e os demais indicadores foram categorizados em medidas de tendência central e dispersão. Por fim, os dados obtidos foram inseridos em uma planilha do software Excel versão 2016 (Microsoft Corp., Estados Unidos). Foram realizados os testes qui-quadrado para análise do tipo de organização de equipe de saúde bucal com os indicadores de atividades coletivas em saúde e o teste de Kruskal-Wallis para as outras variáveis, considerando-se o intervalo de confiança de $95 \%, \mathrm{p}<0,05$. Todos os testes estatísticos foram realizados através do software Statistic Package for the Social Sciences - SPSS versão 13.0 (SPSS Inc., Estados Unidos).

A pesquisa seguiu as recomendações da Resolução $n^{\circ}$ 466/2012 do Conselho Nacional de Saúde, relativa à pesquisa em seres humanos, sendo aprovada pelo Comitê de Ética em Pesquisa com Seres Humanos (CEP) sob parecer $n^{\circ} 3.159 .947$.

\section{Resultados}

Participaram do estudo 166 eSB, sendo 139 de USF, 27 de Upinhas e 9 de UBT. Dessa forma, foram excluídas 5 eSB de USF, 1 eSB de Upinha e 12 UBT, por não possuírem informações registradas no Sisab ou não possuírem cirurgião-dentista registrado no CNES.

Para o indicador de cobertura de primeira consulta odontológica programática, observa-se que as UBT apresentaram os maiores valores de mediana ( $31,8 \%)$, enquanto as Upinhas obtiveram o menor valor $(5,4 \%)$ $(\mathrm{p}=0,000)$ (tabela 1).

Com relação à proporção de exodontia entre procedimentos clínicos odontológicos individuais selecionados, verifica-se que algumas unidades, no ano de 2018, não realizaram extração dentária. Por outro lado, algumas realizam exodontias em excesso, sendo os valores máximos encontrados em USF e UBT, com $32,7 \%$ e $31,6 \%$, respectivamente (tabela 1 ).

O indicador Média de procedimentos odontológicos básicos individuais demonstra quantidade de procedimentos per capita realizados em um determinado período. Nesse caso, as Upinhas apresentaram uma baixa mediana, isto é, metade das equipes realizaram em média menos de um procedimento $(0,3)$ por paciente coberto pela equipe (tabela 1 ). 
Tabela 1. Indicadores de saúde bucal por tipo de organização das equipes de Saúde Bucal da Atenção Básica em Recife (PE), 2018

\begin{tabular}{|c|c|c|c|c|c|c|c|}
\hline \multirow[b]{2}{*}{ Indicadores } & \multicolumn{7}{|l|}{ Tipo de } \\
\hline & Organização & Média & Mediana & Desvio Padrão & Mínimo & Máximo & Valor-pa \\
\hline \multirow{3}{*}{$\begin{array}{l}\text { Cobertura de primeira } \\
\text { consulta odontológica } \\
\text { programática (\%) }\end{array}$} & USF & 11,4 & 9,6 & 7,7 & 0 & 38 & $0,000^{b}$ \\
\hline & Upinha & 7,3 & 5,4 & 7,9 & 0,2 & 42,1 & \\
\hline & UBT & 33,5 & 31,8 & 26,3 & 2,1 & 88,1 & \\
\hline \multirow{3}{*}{$\begin{array}{l}\text { Proporção de exodontia en- } \\
\text { tre procedimentos clínicos } \\
\text { odontológicos individuais } \\
\text { selecionados (\%) }\end{array}$} & USF & 9,4 & 8,2 & 7,1 & 0 & 32,7 & 0,703 \\
\hline & Upinha & 8,1 & 7,5 & 5,3 & 0 & 19,1 & \\
\hline & UBT & 10,3 & 7,5 & 12,3 & 0 & 31,6 & \\
\hline \multirow{3}{*}{$\begin{array}{l}\text { Média de procedimentos } \\
\text { odontológicos básicos } \\
\text { individuais per capita }\end{array}$} & USF & 0,5 & 0,4 & 0,3 & 0,0 & 1,4 & 0,127 \\
\hline & Upinha & 0,4 & 0,3 & 0,4 & 0,0 & 1,6 & \\
\hline & UBT & 0,8 & 0,6 & 0,7 & 0,1 & 2,1 & \\
\hline
\end{tabular}

Fonte: Elaboração própria com dados SIA/SUS28 e Sisab 29 (2018)

a Valor-p calculado pelo Teste estatístico de Kruskal-Wallis; b significância estatística $(p<0,05)$.

Quanto às atividades coletivas, no tocante à realização da ação coletiva de escovação dental supervisionada, as USF realizam mais essa atividade do que as demais unidades. O mesmo resultado é observado para a ação coletiva de aplicação tópica de flúor (tabela 2).

Tabela 2. Atividades coletivas em saúde bucal por tipo de organização das equipes de Saúde Bucal da Atenção Básica em Recife (PE), 2018

\begin{tabular}{|c|c|c|c|c|c|}
\hline \multirow[b]{2}{*}{ Indicador/Tipo de organização } & \multicolumn{2}{|c|}{ Sim } & \multicolumn{2}{|c|}{ Não } & \multirow[b]{2}{*}{ Valor-pa } \\
\hline & N & $\%$ & $\mathbf{N}$ & $\%$ & \\
\hline \multicolumn{6}{|c|}{ Ação Coletiva Escovação Supervisionada } \\
\hline USF & 63 & 45,3 & 76 & 54,7 & \multirow[t]{3}{*}{0,082} \\
\hline Upinha & 9 & 33,3 & 18 & 66,7 & \\
\hline UBT & 1 & 11,1 & 8 & 88,9 & \\
\hline \multicolumn{6}{|c|}{ Ação Coletiva Aplicação Tópica de Flúor } \\
\hline USF & 62 & 44,6 & 77 & 55,4 & \multirow[t]{3}{*}{0,174} \\
\hline Upinha & 8 & 29,6 & 19 & 70,4 & \\
\hline UBT & 2 & 22,2 & 7 & 77,8 & \\
\hline
\end{tabular}

Fonte: Elaboração própria com dados SIA/SUS28 e Sisab29 (2018).

a Valor p calculado pelo Teste qui-quadrado. 


\section{Discussão}

O estudo, realizado no ano de 2018, em Recife, apontou que a forma de organização dos serviços de saúde está associada à cobertura de primeira consulta odontológica programática, sendo maior nas unidades básicas de saúde do modelo tradicional. Para os demais indicadores, não houve associação, demonstrando-se uma similaridade na organização e na oferta de cuidado dos três tipos de organização em saúde bucal analisados.

Para o indicador de primeira consulta odontológica programática, observa-se que há diferença entre ser coberto pela ESF ou não, resultado corroborado por Rocha e Goes ${ }^{27}$, que indicam que usuários de áreas não cobertas têm 1,5 vezes mais chance de acesso. Nesse sentido, o presente trabalho, ao comparar tipo de organização das equipes, também compara áreas cobertas e descobertas pela ESF, visto que são características das USF/Upinhas e UBT, respectivamente.

O modelo de atenção da ESF representa um desafio positivo para os profissionais, na medida em que exige a ruptura com o modelo tecnicista vigente na odontologia tradicional ${ }^{30}$. Assim, sugere-se que o melhor desempenho para as UBT no indicador de cobertura de primeira consulta odontológica programática pode ser resultado da persistência da ênfase em procedimentos, característica do modelo da odontologia tradicional. Esse modelo, apesar de garantir o acesso, não considera a continuidade e a conclusão do tratamento, visto que a literatura aponta que profissionais das eSB com formação em saúde da família apresentam mais chances de concluir o tratamento dos usuários ${ }^{31}$.

As eSB vinculadas à ESF apresentam-se mais em conformidade com os atributos da $\mathrm{AB}$, como o primeiro contato, coordenação do cuidado e integralidade ${ }^{32}$. Além disso, a literatura aponta melhor desempenho na resolutividade, ou seja, na razão entre primeiras consultas odontológicas programáticas e tratamentos concluídos, ao comparar a ESF com
UBS do modelo tradicional18, podendo esse desempenho variar de acordo com o Índice de Desenvolvimento Humano (IDH), sendo maior em municípios de menor IDH $^{33}$.

Estudo realizado em Pernambuco aponta influência da equiparação entre eSF e eSB em indicadores de saúde bucal, onde a cobertura de primeira consulta odontológica programática se mostrou duas vezes maior em municípios com equiparação das equipes, quando comparados aos municípios sem equiparação ${ }^{34}$. Tal evidência vai de encontro aos achados deste estudo, visto que Upinhas, unidades com equiparação entre eSF e eSB, apresentaram os menores valores desse indicador, demonstrando que a ampliação da oferta do serviço nem sempre vem vinculada a uma reorientação do modelo de atenção.

Apesar da semelhança de resultados da proporção de exodontias entre procedimentos clínicos por tipo de organização, as USF apresentaram os maiores valores, resultado evidenciado em outros estudos ao comparar a relação de exodontias apenas com procedimentos restauradores ${ }^{19}$ e a cobertura de exodontia ${ }^{35}$. Entretanto, uma mudança no perfil dos procedimentos de saúde bucal da $\mathrm{AB}$ pode ser verificada nos últimos anos, visto que dados apontam uma predominância de procedimentos restauradores em relação aos cirúrgicos ao longo dos anos ${ }^{36}$. Todavia, devem ser levados em consideração a necessidade da população e os aspectos relativos ao acesso ao tratamento especializado de endodontia, que interferem na escolha do profissional e do paciente acerca da manutenção do elemento dentário, pois fatores locais são determinantes para os resultados dos indicadores ${ }^{35}$.

O estudo aponta que algumas UBS não registraram procedimentos cirúrgicos nos SIS no período investigado. Apesar de o estudo não se propor a fazer uma avaliação qualitativa dos procedimentos realizados na $\mathrm{AB}$, levantam-se algumas hipóteses acerca desses dados. Assim, os cirurgiões-dentistas podem impor algumas limitações de procedimentos realizados, devido à sobrecarga no sistema musculoesquelético, 
que são consequência de posturas não naturais e constantes ${ }^{37}$. Além disso, sugere-se que alguns profissionais não realizam procedimentos cirúrgicos sem exames complementares, visto que estudos apontam que apenas 10,8\% das eSB de Pernambuco apresentam aparelhos radiográficos ${ }^{38}$ e que o tempo de espera para a radiologia no Nordeste é de 1 ano ou mais para $26,2 \%$ das eSB ${ }^{39}$. Recomenda-se o aprofundamento de estudos para além da quantidade de procedimentos realizados, voltados para a qualidade do cuidado ofertado em saúde bucal nas UBS do País.

O indicador média de procedimentos odontológicos básicos individuais per capita na população apontou maiores valores nas UBT, apesar de apresentar menos de um procedimento por paciente no ano de 2018 em todos os tipos de organização. Dutra et al. ${ }^{40}$ apontam resultado oposto. Segundo seus achados, a produção ambulatorial na $\mathrm{AB}$ per capita apresentou valores menores entre a população não cadastrada pela ESF. Tal diferença pode ser resultante do baixo número de procedimentos incluídos no cálculo do indicador, o que pode ter limitado o consolidado de procedimentos realizados no período.

As atividades coletivas realizadas pela $\mathrm{AB}$ são ferramentas fundamentais de promoção da saúde, sendo importante evidenciar a realização dessas atividades por tipo de organização das eSB diante das diferenças estruturais, de processo de trabalho e de carga horária. Neste estudo, percebe-se o papel relevante da ESF na realização das atividades coletivas, o que é corroborado por Pereira et al. ${ }^{35}$ e por Magri et al. ${ }^{19}$, que obtiveram melhores resultados na comparação entre procedimentos preventivos e curativos para USF. Entretanto, no Nordeste do Brasil, ainda predomina um maior número de procedimentos curativos quando comparados aos preventivos ${ }^{36}$. Dessa forma, as atividades de promoção e prevenção à saúde permanecem um desafio para todos os modelos de AB.

Por outro lado, Viana et al. ${ }^{41}$ abordam que a média de escovação dental supervisionada pode estar relacionada com o processo de trabalho das equipes, sendo melhor para padrões de processo de trabalho considerados satisfatórios pelo Programa Nacional de Melhoria do Acesso e da Qualidade da Atenção Básica (PMAQ-AB). Assim, Lima et al. ${ }^{15}$ enfatizam a necessidade de implementação de ações de prevenção e promoção de saúde, mas também de aumento da resolutividade de ações curativas, de forma a superar os desafios relacionados ao cuidado integral dos pacientes. Portanto, a ESF deve demandar ações que respondam às necessidades de saúde da população, valorizando o equilíbrio entre ações curativas e preventivas. O processo de trabalho mostra-se como um fator a ser considerado. Além disso, estudos apontam poucos profissionais qualificados na área de atuação da saúde coletiva, refletindo o modelo curativista, com foco hospitalocêntrico ${ }^{14}$ e que é um obstáculo para a efetividade e o potencial das ações coletivas de atingirem a população ${ }^{19}$.

No entanto, a análise da $\mathrm{AB}$ revela limitações nos três tipos de organização de eSB estudados: as UBT apresentaram maiores resultados nos indicadores de cobertura de primeira consulta odontológica programática e média de procedimentos odontológicos básicos individuais; as Upinhas realizaram menos procedimentos de exodontia; e as USF tiverem resultados mais favoráveis com relação às atividades coletivas.

Por se tratar de dados secundários, obtidos nos sistemas de informação, podem ocorrer possíveis falhas no registro dos procedimentos realizados. Assim, os resultados devem orientar a avaliação dentro de um contexto e agregar informações oriundas de outras fontes de informação. Outra limitação foi a baixa quantidade de usuários cadastrados em cada UBS, que fez com que, em boa parte das unidades, o cálculo dos indicadores fosse realizado a partir de um valor populacional estimado. Entretanto, este estudo evidencia lacunas que são fundamentais ao monitoramento e à avaliação local, que podem ser ferramentas para a gestão impulsionar a busca de estratégias 
de melhoria e realização de novos estudos comparativos.

\section{Considerações finais}

O estudo observou que, no ano de 2018, na cidade de Recife, as unidades básicas tradicionais apresentaram melhores resultados nos indicadores relacionados ao acesso ao tratamento clínico, e as unidades de saúde da família estiveram mais voltadas para ações coletivas de prevenção. Dessa forma, os resultados demonstram a necessidade de discussões em nível local acerca do desempenho de cada Unidade Básica de Saúde, a fim de que, juntamente com os atores envolvidos, seja induzida uma requalificação do processo de trabalho de modo a fortalecer a $\mathrm{AB}$ e o cuidado pautado na longitudinalidade e na resolutividade.

\section{Colaboradores}

Menezes LS (0000-0001-6049-7555)* e Lucena EHG (0000-0003-3431-115X)* contribuíram substancialmente para a concepção, o planejamento, a análise e a interpretação dos dados, elaboração do rascunho ou revisão crítica do conteúdo. Figueiredo N (0000-00016181-8728)* contribuiu substancialmente para a concepção e o planejamento, elaboração do rascunho ou revisão crítica do conteúdo. Cavalcanti YW (0000-0002-3570-9904)* contribuiu substancialmente para a análise e a interpretação dos dados. Ceissler CAS (0000-0002-4077-9245)* contribuiu substancialmente para a análise e a interpretação dos dados, elaboração do rascunho ou revisão crítica do conteúdo. Todos os autores e autoras participaram da aprovação da versão final do manuscrito. 


\section{Referências}

1. Starfield B. Atenção primária: equilíbrio entre necessidades de saúde, serviços e tecnologia. Brasília, DF: Organização das Nações Unidas para a Educação, a Ciência e a Cultura; Ministério da Saúde; 2002.

2. Giovanella L. Atenção Básica ou atenção primária à saúde? Cad. Saúde Pública. 2018; 34(8):e00029818.

3. Brasil. Ministério da Saúde. Programa de Saúde da Família. Brasília, DF: Cosac; 1994.

4. Brasil. Ministério da Saúde. Portaria n ${ }^{\circ} 2.436$, de 21 de setembro de 2017. Aprova a Política Nacional de Atenção Básica, estabelecendo a revisão de diretrizes para a organização da Atenção Básica, no âmbito do Sistema Único de Saúde (SUS). Diário Oficial da União. 22 Set 2017.

5. Morosini MVGC, Fonseca AF, Lima LD. Política Nacional de Atenção Básica 2017: retrocessos e riscos para o Sistema Único de Saúde. Saúde debate. 2018; 42(116):11-24.

6. Brasil. Ministério da Saúde. Portaria $n^{0} 1.444,28$ de dezembro de 2000. Estabelece incentivo financeiro para reorganização da saúde bucal prestada nos municípios por meio do Programa Saúde da Família. Diário Oficial da União. 29 Dez 2000.

7. Lucena EHG, Pucca Junior GA, Sousa MF. A Política Nacional de Saúde Bucal no Brasil no contexto do Sistema Único de Saúde. Tempus actas de saúde colet. 2011; 5(3):53-63.

8. Narvai PC. Avanços e Desafios da Política Nacional de Saúde Bucal no Brasil. Tempus actas de saúde colet. 2011; 5(3):21-34.

9. Mattos GCM, Ferreira EF, Leite ICG, et al. A inclusão da Equipe de Saúde Bucal na Estratégia Saúde da Família: entraves, avanços e desafios. Ciênc. Saúde Colet. 2014; 19(2):373-382.

10. Brasil. Ministério da Saúde, Secretaria de Atenção à Saúde. Diretrizes da Política Nacional de Saúde Bucal [internet]. Brasília, DF: MS; 2004. [acesso em 2020 jun 18]. Disponível em: http://189.28.128.100/dab/ docs/publicacoes/geral/diretrizes_da_politica_nacional_de_saude_bucal.pdf.

11. Brasil. Secretaria de Atenção Primária à Saúde. Sala de Apoio à Gestão Estratégica [internet]. [acesso em 2020 jun 18]. Disponível em: http://sage.saude.gov. br/\#.

12. Silva SF, Martelli PJL, Sá DA, et al. Análise do avanço das equipes de saúde bucal inseridas na Estratégia Saúde da Família em Pernambuco, região Nordeste, Brasil, 2002 a 2005. Ciênc. Saúde Colet. 2011; 1(16):211-20.

13. Souza TMS, Roncalli AG. Saúde bucal no Programa Saúde da Família: uma avaliação do modelo assistencial. Cad. Saúde Pública. 2007; (23):2727-39.

14. Martelli PJL, Cabral APS, Pimentel FC, et al. Análise do modelo de atenção à saúde bucal em municípios do estado de Pernambuco. Ciênc. Saúde Colet. 2008; 13(5):1669-74.

15. Lima VT, Codato AB, Higasi MS, et al. Percepções de cirurgiões-dentistas sobre o trabalho no Programa Saúde da Família. Rev da ABENO. 2018; 18(4):130139.

16. Lima KWS, Antunes JLF, Silva ZP. Percepção dos gestores sobre o uso de indicadores nos serviços de saúde. Saúde Soc. 2015; 24(1):61-71.

17. Rede Interagencial de Informação para a Saúde. Indicadores básicos para a saúde no Brasil: conceitos e aplicações. 2. ed. Brasília, DF: Ripsa; Opas; 2008.

18. Bulgareli J, Cortellazzi KL, Ambrosano GMB, et al. A resolutividade em saúde bucal na Atenção Básica como instrumento para avaliação dos modelos de atenção. Ciênc. Saúde Colet. 2014; 19(2):383-91.

19. Magri VL, Aciole GG, Salomão DGF, et al. Estudo comparativo de indicadores de saúde bucal em mu- 
nicípio do estado de São Paulo. Saúde debate. 2016; 40(108):144-55.

20. Recife. Prefeitura. Construção de Upinha Dia inaugura novo padrão de atendimento na saúde [internet]. [Recife, PE]: 2013. [acesso em 2019 out 15]. Disponível em: http://www2.recife.pe.gov.br/noticias/23/08/2013/ construcao-de-upinha-dia-inaugura-novo-padrao-de-atendimento-na-saude.

21. Brasil. Ministério da Saúde. Sistema de Gerenciamento da Tabela de Procedimentos, medicamentos e OPM do SUS - SIGTAP [internet]. [acesso em 2018 nov 15]. Disponível em: http://sigtap.datasus.gov.br/ tabela-unificada/app/sec/inicio.jsp.

22. Brasil. Ministério da Saúde, Secretaria de Atenção à Saúde, Departamento de Atenção Básica. Programa Nacional de Melhoria do Acesso e da Qualidade da Atenção Básica (PMAQ): Manual Instrutivo para as Equipes. Brasília, DF: Ministério da Saúde; 2017.

23. Silva LS, Santana KR, Pinheiro HHC, et al. Indicadores de Atenção Básica e especializada em saúde bucal nos municípios do Estado do Pará, Brasil: estudo ecológico, 2001-2010. Epidemiol Serv Saúde. 2013; 22(2):325-334.

24. Brasil. Ministério da Saúde. Índice de desempenho do SUS - IDSUS [internet]. [acesso em 2018 nov 15]. Disponível em: http://idsus.saude.gov.br/assets/detalhadas.pdf.

25. Brasil. Ministério da Saúde. Cadernos de Atenção Básica ${ }^{\circ}$ 17. Série A. Normas e Manuais Técnicos. Brasília, DF: Ministério da Saúde; 2008.

26. Campos ACV, Borges CM, Leles CR, et al. Indicadores socioeconômicos e de saúde da Atenção Básica nos municípios da região metropolitana de Belo Horizonte. Arq Odontol. 2012; 48(1):6-12.

27. Rocha RACP, Goes PSA. Comparação do acesso aos serviços de saúde bucal em áreas cobertas e não cobertas pela Estratégia Saúde da Família em Campina Grande, Paraíba, Brasil. Cad. Saúde Pública. 2008; 24(12):2871-80
28. Brasil. Ministério da Saúde. Sistemas de Informações Ambulatoriais do SUS (SIA/SUS). [internet]. [acesso em 2019 abr 10]. Disponível em: http://sia.datasus. gov.br/principal/index.php.

29. Brasil. Ministério da Saúde. Sistema de Informação em Saúde para a Atenção Básica (SISAB). [internet]. [acesso em 2019 abr 10]. Disponível em: https://sisab.saude.gov.br/.

30. Leme PAT, Bastos RA, Turano ER, et al. A clínica do dentista na Estratégia Saúde da Família: entre a inovação e o conservadorismo. Physis. 2019 [acesso em 2020 jun 18]; 29(1):1-19. Disponível em: http://www. scielo.br/scielo.php?script=sci_arttext\&pid=S0103$-73312019000100610 \& \operatorname{lng}=\mathrm{en}$.

31. Santos NML, Hugo FN. Formação em Saúde da Família e sua associação com processos de trabalho das Equipes de Saúde Bucal da Atenção Básica. Ciênc. Saúde Colet. [internet]. 2018 [acesso em 2020 jun 18]; 23(12):4319-4329. Disponível em: http://dx.doi. org/10.1590/1413-812320182312.12922016.

32. Fagundes DM, Thomaz EBAF, Queiroz RCS, et al. Diálogos sobre o processo de trabalho em saúde bucal no Brasil: uma análise com base no PMAQ-AB. Cad. Saúde Pública. 2018; 34(9):e00049817.

33. Fernandes JKB, Pinho JRO, Queiroz RCS, et al. Avaliação dos indicadores de saúde bucal no Brasil: tendência evolutiva pró-equidade? Cad. Saúde Pública. 2016; 32(2):1-18.

34. Pimentel FC, Albuquerque PC, Martelli PJL, et al. Análise dos indicadores de saúde bucal do Estado de Pernambuco: desempenho dos municípios segundo porte populacional, população cadastrada no sistema de informação da Atenção Básica e proporção na Estratégia Saúde da Família. Cad. Saúde Colet. 2014; 22(1):54-61.

35. Pereira CRS, Roncalli AG, Cangussu MCT, et al. Impacto da Estratégia Saúde da Família sobre indicadores de saúde bucal: análise em municípios do Nordeste brasileiro com mais de 100 mil habitantes. Cad. Saúde Pública. 2012; 28(3):449-462. 
36. Silva SE, Araújo JHP, Laureano ICC, et al. Caracterização do modelo de Atenção Básica à saúde bucal na região nordeste no período de 2015-2017. Arch Health Invest. 2018; 7(10):402-407.

37. Santos MC, Vogt MS. Estudo exploratório dos distúrbios musculoesqueléticos em cirurgiões-dentistas da Associação Brasileira de Odontologia - Regional Missioneira da cidade de Santo Ângelo/RS. Fisioter Bras. 2009; 10(4):229-234.

38. Pimentel FC, Albuquerque PC, Souza WV, et al. A atenção em saúde bucal no estado de Pernambuco: analisando a estrutura e as ações preventivas e curativas, segundo porte populacional. Rev baiana saúde pública [internet]. 2014 [acesso em 2020 jun 22]; 38(2):297-317.

39. Casotti E, Contarato PC, Fonseca ABM, et al. Atenção em Saúde Bucal no Brasil: uma análise a partir da
Avaliação Externa do PMAQ-AB. Saúde debate [internet]. 2014 [acesso em 2020 jun 22]; 38(esp):140157. Disponível em: https://doi.org/10.5935/0103-1104.2014S011. ISSN 0103-1104.

40. Dutra ILS, Reis WFCA, Ferreira MC. Informação em Saúde Bucal: a realidade sanitária de um município do Nordeste brasileiro. Re Saúd Digi Tec Edu. 2018; (3):32-42.

41. Viana IB, Moreira RS, Martelli PJL, et al. Avaliação da qualidade da assistência em saúde bucal na Atenção Primária à Saúde em Pernambuco, 2014. Epidemiol Serv Saúde. 2019; 28(2):e2018060.

Recebido em 03/03/2020

Aprovado em 13/08/2020

Conflito de interesses: inexistente

Suporte financeiro: não houve 